\title{
On gravitational interaction of fermions
}

\author{
Yuri N. Obukhov \\ Department of Theoretical Physics \\ Moscow State University \\ 117234 Moscow, Russia
}

\begin{abstract}
We discuss some aspects of the gravitational interaction of the relativistic quantum particles with spin $1 / 2$. The exact Foldy-Wouthuysen transformation is constructed for the Dirac particle coupled to the static spacetime metric. The quasirelativistic limit of the theory is then analyzed. Using the analogous method, we obtain the exact Cini-Touschek transformation and discuss the ultra-relativistic limit of the fermion theory. We show that the Foldy-Wouthuysen transformation is not uniquely defined, and the corresponding ambiguity is deeply rooted in the relativistic quantum theory.
\end{abstract}

\section{Introduction}

The recent development of experimental technique, in particular of the neutron interferometric methods [1], has provided the first direct tests of the interaction of the quantum spinless particles with the classical gravitational field. There is a little doubt that a further technological progress (using the polarized neutrons, atomic interferometers, etc) will soon make it possible to measure the higher order gravitational and inertial effects of the quantum particle with spin. Theoretical studies of the relativistic quantum theory in a curved spacetime have predicted a number of interesting manifestations of the spingravity coupling for the Dirac fermion, see [2, 12, 13, 9], e.g. In most cases, the various approximate schemes were used for the case of the weak gravitational field. Here the exact results for an arbitrary static spacetime geometry are reported.

A massive quantum particle with spin $1 / 2$ is described by the relativistic Dirac theory. In the curved spacetime, the fermion wave function - 4-spinor field $\psi$ - satisfies the covariant Dirac equation

$$
\left(i \hbar \gamma^{\alpha} D_{\alpha}-m c\right) \psi=0 .
$$

The spinor covariant derivative is defined by

$$
D_{\alpha}=h_{\alpha}^{i} D_{i}, \quad D_{i}:=\partial_{i}+\frac{i}{4} \widehat{\sigma}_{\alpha \beta} \Gamma_{i}^{\alpha \beta},
$$

which shows that the gravitational and inertial effects are encoded in the coframe (vierbein) and the Lorentz connection coefficients $h_{i}{ }^{\alpha}, \Gamma_{i}{ }^{\alpha \beta}=-\Gamma_{i}{ }^{\beta \alpha}$. We use the Greek alphabet for the indices which label the components with respect to a local Lorentz frame $e_{\alpha}=h_{\alpha}^{i} \partial_{i}$, whereas the Latin indices refer to the local spacetime coordinates $x^{i}$. For the 
Dirac matrices, the conventions of [3] are used. In particular, we have $\beta=\gamma^{\widehat{0}}, \vec{\alpha}=\beta \vec{\gamma}$, $\gamma_{5}=-i \gamma^{\widehat{0}} \gamma^{\widehat{1}} \gamma^{\widehat{2}} \gamma^{3}, \widehat{\sigma}^{\alpha \beta}=i \gamma^{[\alpha} \gamma^{\beta]}$. The spin matrix is defined by $\vec{\Sigma}=i \vec{\gamma} \times \vec{\gamma} / 2=-\gamma_{5} \vec{\alpha}$.

Let us consider the metric of the static spacetime

$$
d s^{2}=V^{2}\left(d x^{0}\right)^{2}-W^{2}(d \vec{x} \cdot d \vec{x}) .
$$

Here $x^{0}=c t$, and $V=V(\vec{x}), W=W(\vec{x})$ are arbitrary functions of $\vec{x}$. Many important particular cases belong to this family: (i) the flat Minkowski spacetime in accelerated frame corresponds to the choice $V=1+(\vec{a} \cdot \vec{x}) / c^{2}, W=1$, (ii) Schwarzschild spacetime in the isotropic coordinates with $r:=\sqrt{\vec{x} \cdot \vec{x}}$ is obtained for $V=\left(1-\frac{G M}{2 c^{2} r}\right)\left(1+\frac{G M}{2 c^{2} r}\right)^{-1}, W=$ $\left(1+\frac{G M}{2 c^{2} r}\right)^{2}$, (iii) de Sitter spacetime in static frame is recovered for $V=\frac{1+r^{2} / \ell^{2}}{1-r^{2} / \ell^{2}}, W=$ $2 /\left(1-r^{2} / \ell^{2}\right)$, where $\ell$ is the constant curvature radius (with the curvature 2 -form $R^{\alpha \beta}=$ $\left.1 / \ell^{2} \vartheta^{\alpha} \wedge \vartheta^{\beta}\right)$, (iv) the product spacetime $R \times S^{3}$ (fermion on a sphere) arises when $V=$ $1, W=\left(1+r^{2} /\left(4 L^{2}\right)\right)^{-1}$ with $L$ radius of the sphere $S^{3}$.

Using (3), one can bring the Dirac equation (1) to the Schrödinger form

$$
i \hbar \frac{\partial \psi}{\partial t}=\widehat{\mathcal{H}} \psi
$$

with the Hamilton operator

$$
\widehat{\mathcal{H}}=\beta m c^{2} V+\frac{c}{2}[(\vec{\alpha} \cdot \vec{p}) \mathcal{F}+\mathcal{F}(\vec{\alpha} \cdot \vec{p})] .
$$

Here we introduced $\mathcal{F}=V / W$.

\section{Foldy-Wouthuysen transformation}

In order to reveal the true physical content of the theory and to find its correct interpretation, one needs to perform the Foldy-Wouthuysen (FW) transformation [4]. Technically, this yields the representation in which the quantum states with positive and negative energy become uncoupled.

We use here the approach of Eriksen [5] to construct the exact Foldy-Wouthuysen transformation. The energy sign operator (Pauli) is defined by $\widehat{\Lambda}=\widehat{\mathcal{H}} / \sqrt{\widehat{\mathcal{H}}^{2}}$. It is Hermitian, unitary, and idempotent: $\widehat{\Lambda}^{2}=\widehat{\Lambda}^{\dagger} \widehat{\Lambda}=1$. The unitary operator $U$ which maps the Dirac representation to the FW-representation

$$
\psi \longrightarrow \psi^{F}=U \psi
$$

should satisfy the condition

$$
U \widehat{\Lambda} U^{\dagger}=\beta .
$$

Remarkably, for our case, the exact FW-transformation exists. Consider the operator

$$
J:=i \gamma_{5} \beta=\left(\begin{array}{cc}
0 & i \\
-i & 0
\end{array}\right)
$$

It is Hermitian, $J^{\dagger}=J$, unitary, and idempotent: $J J^{\dagger}=J^{2}=1$, and it anticommutes both with the Hamiltonian, and with $\beta$ :

$$
J \widehat{\mathcal{H}}+\widehat{\mathcal{H}} J=0, \quad J \beta+\beta J=0 .
$$


Then the FW transformation (7) is realized by

$$
U=\frac{1}{2}(1+\beta J)(1+J \widehat{\Lambda})
$$

and the corresponding FW Hamiltonian reads

$$
\widehat{\mathcal{H}}^{F}=U \widehat{\mathcal{H}} U^{\dagger}=\left[\sqrt{\widehat{\mathcal{H}^{2}}}\right] \beta+\left\{\sqrt{\widehat{\mathcal{H}}^{2}}\right\} J
$$

As usual, even and odd parts of an operator $Q$ are defined by $[Q]:=\frac{1}{2}(Q+\beta Q \beta)$ and $\{Q\}:=\frac{1}{2}(Q-\beta Q \beta)$. Explicitly, we have for the square of (5)

$$
\widehat{\mathcal{H}}^{2}=m^{2} c^{4} V^{2}+\mathcal{F} c^{2} p^{2} \mathcal{F}+\frac{\hbar^{2} c^{2}}{2}\left(\mathcal{F} \vec{\nabla} \vec{f}-\vec{f}^{2} / 2\right)+\hbar c^{2} \mathcal{F} \vec{\Sigma} \cdot(\vec{f} \times \vec{p}+J m c \vec{\phi})
$$

which contains the odd piece (the last term). Here: $\vec{\phi}:=\vec{\nabla} V, \vec{f}:=\vec{\nabla} \mathcal{F}$.

The obtained FW Hamiltonian is exact. However, for most practical purposes, it is sufficient to consider the non-relativistic limit, and use the quasi-relativistic wave functions, treating all the interaction terms as perturbations. The quasi-relativistic approximation is straightforwardly obtained by assuming that $m c^{2}$ term is dominating, and expanding $\sqrt{\widehat{\mathcal{H}}^{2}}$ in powers of $1 /\left(m c^{2}\right)$. However, the massless case is covered only by the exact result.

Expanding $\sqrt{\widehat{\mathcal{H}}^{2}}$ in powers of $1 /\left(m c^{2}\right)$, we find finally the quasi-relativistic Hamiltonian:

$$
\begin{aligned}
\widehat{\mathcal{H}}^{F} \approx & \underbrace{\beta m c^{2} V}_{\text {COW }}+\underbrace{\frac{1}{4 m} \beta\left(\frac{1}{W} p^{2} \mathcal{F}+\mathcal{F} p^{2} \frac{1}{W}\right)}_{\text {kinetic rel. red shift }}+\underbrace{\frac{\hbar^{2}}{4 m W} \beta(\triangle \mathcal{F})}_{\text {grav. Darwin term }}-\frac{\hbar^{2}}{8 m V} \beta \vec{f}^{2} \\
& +\underbrace{\frac{\hbar}{4 m} \beta \vec{\Sigma} \cdot\left(\frac{1}{W} \vec{f} \times \vec{p}+\vec{f} \times \vec{p} \frac{1}{W}\right)}_{\text {grav/inert. spin - orbit. term }}+\frac{\hbar c}{2 W}(\vec{\Sigma} \cdot \vec{\phi}) .
\end{aligned}
$$

The first two terms describe the familiar effects already measured experimentally for spinless particles (Colella-Overhauser-Werner and Bonse-Wroblewski, [1]). The first term in the second line represents the new inertial/gravitational spin-orbital momentum effects, cf. 112, 13. The "gravitational Darwin" term admits a physical interpretation similar to usual electromagnetic Darwin term, reflecting the zitterbewegung fluctuation of the fermion's position with the mean square $<(\delta r)^{2}>\sim \hbar^{2} /(m c)^{2}$.

It is interesting to observe the emergence of the spin-gravitational moment coupling which is described by the last term in (13). Such interaction was predicted, in a phenomenological approach, by Kobzarev and Okun [6] and was discussed by Peres [7], see also the recent reviews [8]. The presence of this term demonstrates the validity of the equivalence principle for the Dirac fermions [9].

\section{Cini-Touschek transformation}

In the study of the high-energy neutrino effects in the gravitational field of a massive compact object (see [10], e.g.), it is convenient to use a different representation which is directly related to the ultra-relativistic rather than to the quasi-relativistic limit. The new representation is in this sense complementary to the FW picture. 
The corresponding limit (when $m c^{2} \ll c|p|$ ) is achieved with the help of the CiniTouschek (CT) transformation [11]. We can construct the exact CT-transformation for a fermion in the static metric (3) using the scheme similar to the above FW case. To begin with, we observe that the operator

$$
\widehat{\mathcal{P}}=\frac{\vec{\alpha} \cdot \vec{p}}{|p|}
$$

is Hermitian, unitary, and idempotent, $\widehat{\mathcal{P}}^{2}=1$. It is proportional to the chirality operator $\hat{\chi}=\vec{\Sigma} \cdot \vec{p} /|p|=-\gamma_{5} \widehat{\mathcal{P}}$. Evidently, we have

$$
\widehat{\mathcal{P}} J+J \widehat{\mathcal{P}}=0 .
$$

In complete analogy to (7), the CT-transformation is determined by the unitary operator $U$ which satisfies

$$
U \widehat{\Lambda} U^{\dagger}=\widehat{\mathcal{P}} .
$$

Thus, technically, we need to replace $\beta \leftrightarrow \widehat{\mathcal{P}}$ everywhere in the above derivations. The explicit CT-operator then reads

$$
U=\frac{1}{2}(1+\widehat{\mathcal{P}} J)(1+J \widehat{\Lambda})
$$

and the Cini-Touschek Hamiltonian is

$$
\widehat{\mathcal{H}}^{C T}=\left[\sqrt{\widehat{\mathcal{H}^{2}}}\right]^{\mathcal{P}} \widehat{\mathcal{P}}+\left\{\sqrt{\widehat{\mathcal{H}^{2}}}\right\}^{\mathcal{P}} J .
$$

Here, the " $\widehat{\mathcal{P}}$-odd/even" parts of an operator are defined by the same token as the usual " $\beta$-odd/even" parts. As a simple application, we check that for the free particle (18) yields $\widehat{\mathcal{H}}^{C T}=E \widehat{\mathcal{P}} \approx c \vec{\alpha} \cdot \vec{p}$ which is the correct ultra-relativistic Hamiltonian.

\section{Ambiguities}

The presence of the spin-gravitational moment in the quasi-relativistic FW Hamiltonian requires some comments.

FW transformation is defined with a certain ambiguity. Let us consider the unitary transformation $U^{\prime}=e^{i S}$ with

$$
S=\frac{\beta}{m c}\{b(x)(\vec{\Sigma} \vec{p})+(\vec{\Sigma} \vec{p}) b(x)\},
$$

where $b(x)$ is an arbitrary function of the spatial coordinates. The spaces of quantum states with positive and negative energies are invariant under the action of this operator. For the Hamiltonian of the unitary equivalent representation, we find, in a perturbative manner:

$$
\begin{aligned}
\widehat{\mathcal{H}}^{\prime}=U^{\prime} \widehat{\mathcal{H}}^{F} U^{\prime \dagger}= & \widehat{\mathcal{H}}^{F}+2 \hbar c b(\vec{\Sigma} \vec{\phi})+\frac{i \hbar}{m} \beta[b(\vec{\Sigma} \vec{p}),(1 / W+2 b)(\vec{\Sigma} \vec{\phi})] \\
& +\frac{\hbar^{2}}{2 m}(1 / W+2 b) \beta[(\vec{\Sigma} \vec{\nabla} b),(\vec{\Sigma} \vec{\phi})]+\mathcal{O}\left(1 / m^{2}\right) .
\end{aligned}
$$

Using (13), we find that the choice $2 b=-\frac{1}{2 W}$ yields the approximate form of the FW Hamiltonian reported by Fischbach et al [12] and by Hehl and Ni 13. 
The mentioned ambiguity is deeply rooted in the relativistic quantum theory. The FW representation is often treated merely as a rigorous method to derive the quasi-relativistic limit of the Dirac equation (see [14], e.g.), refining the "non-rigorous" derivation based on the direct elimination of the so-called small components of the 4-spinor wave function. And indeed, one can straightforwardly verify that the direct derivation of the Pauli equation suffers from the same ambiguity: Recall that after eliminating the small components, the remaining 2 -spinor wave function should be properly normalized [15]. The corresponding normalization operator is not uniquely defined and this yields the transformation of the type (20) of the quasi-relativistic Hamiltonian.

Furthermore, one can easily find the relevant ambiguities of that kind in the full (relativistic) Dirac theory. For example, the Hamiltonian of the free particle $\widehat{\mathcal{H}}=c(\vec{\alpha} \vec{p})+\beta m c^{2}$ is invariant under the unitary transformation of the wave function described by

$$
U=\sqrt{\frac{E+m c^{2}}{2 E}}\left(1+\frac{i c}{E+m c^{2}}(\vec{\Sigma} \vec{p}) \widehat{\Lambda}\right) .
$$

Operators of position, spin and energy (Hamiltonian) can have different form in the unitary equivalent representations, and one should properly determine them in order to analyze the physical effects. Certainly, the observable quantities measured in experiment do not depend on the choice of representation.

\section{Discussion and conclusion}

Approximate scheme (see Bjorken-Drell [3], e.g.) was developed for the case of electromagnetic coupling. As it is well known, the idea is to remove, order by order in $1 / m$, odd terms from the Hamiltonian $\widehat{\mathcal{H}}=\widehat{\mathcal{H}}_{1}=\beta m c^{2}+\mathcal{E}+\mathcal{O}$. A unitary transformation $\psi_{2}=U_{21} \psi_{1}$, with $U_{21}=e^{i S}$, yields (in the time-independent case) the perturbative construction of the new Hamiltonian

$$
\widehat{\mathcal{H}}_{2}=U_{21} \widehat{\mathcal{H}}_{1} U_{21}^{\dagger}=\widehat{\mathcal{H}}_{1}+\left[i S, \widehat{\mathcal{H}}_{1}\right]+\frac{1}{2}\left[i S,\left[i S, \widehat{\mathcal{H}}_{1}\right]\right]+\frac{1}{3 !}\left[i S,\left[i S,\left[i S, \widehat{\mathcal{H}}_{1}\right]\right]\right]+\ldots
$$

In electrodynamics, the odd $\mathcal{O}$ and even $\mathcal{E}$ parts do not depend on the mass $m$. Instead, they are proportional to electromagnetic charge $e$, and that fact makes the approximate scheme working. For example, choosing at the first step $S=-i \beta \mathcal{O} / 2 m$, we remove the original odd part and find $\widehat{\mathcal{H}}_{2}=\beta m c^{2}+\mathcal{E}^{\prime}+\mathcal{O}^{\prime}$ where the new odd part

$$
\mathcal{O}^{\prime}=\frac{\beta}{2 m}[\mathcal{O}, \mathcal{E}]-\frac{\mathcal{O}^{3}}{3 m^{2}}
$$

has a higher order in $1 / m$ than the new even part. However, for the gravitational/inertial case, $\mathcal{E}$ is proportional to the gravitational/inertial charge $m$. As a result, the new odd term in $\widehat{\mathcal{H}}_{2}$ is of order $m^{0}$. The same happens at every step of the approximate scheme: the "remaining" even terms have the same order in $1 / m$ as the "removed" odd terms. This makes the issue of the convergence of the approximate scheme problematic. In our approach, this deficiency is avoided by using the exact FW transformation.

Here we have demonstrated how to obtain the exact FW and CT transformations in the covariant Dirac theory. The detailed discussion of the corresponding applications to the specific quasi-relativistic and ultra-relativistic physical problems will be presented elsewhere. 
Acknowledgment I would like to thank the Alexander von Humboldt Foundation for the invitation and support.

\section{References}

[1] R. Colella, A.W. Overhauser, and S.A. Werner, Phys. Rev. Lett. 34 (1975) 1472;

U. Bonse and T. Wroblewski, Phys. Rev. Lett. 51 (1983) 1401.

[2] C.G. de Oliveira and J. Tiomno, Nuovo Cim. 24 (1962) 672; J. Audretsch and G. Schäfer, Gen. Rel. Grav. 9 (1978) 243; Y.Q. Cai and G. Papini, Phys. Rev. Lett. 66 (1991) 1259; Phys. Rev. Lett. 68 (1992) 3811; D. Singh and G. Papini, Nuovo Cim. B115 (2000) 223; J.C. Huang, Ann. d. Phys. 3 (1994) 53; K. Varjú and L.H. Ryder, Phys. Lett. A250 (1998) 263; L. Ryder, J. Phys. A: Math. Gen. A31 (1998) 2465. K. Varjú and L.H. Ryder, Phys. Rev. D62 (2000) 024016.

[3] J.D. Bjorken and S.D. Drell, Relativistic Quantum Mechanics (McGraw-Hill: San Francisco, 1964).

[4] L.L. Foldy and S.A. Wouthuysen, Phys. Rev. 78 (1950) 29.

[5] E. Eriksen and M. Kolsrud, Nuovo Cim. 18 (1960) 1; A.G. Nikitin, J. Phys. A: Math. Gen. A31 (1998) 3297.

[6] I.Yu. Kobzarev and L.B. Okun, Sov. Phys. JETP 16 (1963) 1343 [ZhETF 43 (1962) 1904 (in Russian)]; see also J. Leitner and S. Okubo, Phys. Rev. 136 (1964) 1542.

[7] A. Peres, Phys. Rev. D18 (1978) 2739.

[8] B. Mashhoon, Class. Quantum Grav. 17 (2000) 2399; L.H. Ryder and B. Mashhoon, to be published in the Proceedings of the Ninth Marcel Grossmann Meeting (Rome, 2000); e-Print Archive: gr-qc/0102101.

[9] Yu.N. Obukhov, Phys. Rev. Lett. 86 (2001) 192.

[10] K. Konno and M. Kasai, Prog. Theor. Phys. 100 (1998) 1145.

[11] M. Cini and B. Touschek, Nuovo Cim. 7 (1958) 422; S.K. Bose, A. Gamba, and E.C.G. Sudarshan, Phys. Rev. 113 (1959) 1661.

[12] E. Fischbach, B.S. Freeman, and W.-K. Cheng, Phys. Rev. D23 (1981) 2157.

[13] F.W. Hehl and W.-T. Ni, Phys. Rev. D42 (1990) 2045.

[14] M.E. Rose, Relativistic electron theory (John Wiley \& Sons: New York, 1961).

[15] A.I. Akhiezer and V.B. Berestetsky, Quantum electrodynamics (Consultants Bureau: Oak Ridge) [Translation form the Russian edition: Kvantovaya elektrodinamika (GITTL: Moscow, 1953)], see Sec. 14. 